\title{
IRPanet: Intelligent Routing Protocol in VANET for Dynamic Route Optimization
}

\author{
Rafi Ullah ${ }^{1}$, Shah Muhammad Emad ${ }^{2}$, Taha Jilani ${ }^{3}$, Waqas Azam ${ }^{4}$, Muhammad Zain uddin ${ }^{5}$ \\ College of Computing and Information Sciences, PAF-KIET Karachi, Pakistan 1, 2, 3, 5 \\ Solution Archetect and Project Manager, 10Pearls, Karachi, Pakistan ${ }^{4}$
}

\begin{abstract}
This paper presents novel routing protocol, IRPANET (Intelligent Routing Protocol in VANET) for Vehicular Adhoc Network (VANET). Vehicular Ad Hoc Networks are special class of Mobile Adhoc Network, created by road vehicles installed with wireless gadgets). Since the environment is dynamic due to high mobility and the topology changes are too frequent, no connection or path can be established between nodes. The issues are challenging for the design of an effective and efficient protocol for such a dynamic environment. This problem can be solved using probabilistic, heuristic and even machine learning based approaches incorporated with store and forward mechanism. Here, we proposed a design framework using heuristics and probabilistic approaches composite with the time series techniques for selecting best and optimize path for forwarding packets using open street map (OSM). Our proposed algorithm uses various parameters (Heuristics Based Routing) for calculating optimal path for packets to be sent, such geographical position (GPS installed in every vehicle), velocity / speed of vehicle, priority of the packets, distances (Euclidean, Haversine, Vicinity) between vehicle, direction of vehicle, communication range of the vehicle, free buffer of nodes and network congestion. These networks can be used for medical emergency, security, entertainment and routing purposes (applications of VANET). These parameters while used in collaboration provide us a very strong and admissible heuristics. We have mathematically proved that the proposed technique is efficient for the routing of packets especially in medical emergency situation.
\end{abstract}

Keywords-Intelligent routing protocol; heuristics based routing; applications of VANET; Vehicular Adhoc Network; VANET routing protocol

\section{INTRODUCTION}

Mobile ad hoc network usually denoted by MANET can be defined as a network that has many free or autonomous nodes wirelessly connected, composed of mobile devices or mobile objects that can arrange themselves in various ways and operate without strict top-down network administration (infrastructure-less networks and self-configuring networks). These freely movables devices can move in any of the direction specified independently of other nodes, hence results highly complex sceneries such as dynamic topology, autonomous topology, frequent network disconnections, efficient and fast routing of packets and many of the characteristics that make it distinguish from traditional networks. Wireless ad hoc network or ad hoc wireless networks are the alternative terms used for MANET. When vehicles are used as movable objects in MANET, this special class of MANET is known as Vehicular Adhoc Networks usually known as VANETs [1] [2]. VANET Communication usually take place between vehicle to vehicle or vehicle to roadside unit (like base station, stationary towers etc.) [3]. In VANET the high speed nodes (vehicles having speed generally from $40 \mathrm{~km} / \mathrm{hr}$. to $180 \mathrm{~km} / \mathrm{hr}$.) can be used as transmitters and receivers. Communication environment is dynamic and it changes constantly with time. Nodes are carrying a lot of battery power with itself along with autorecharge facility hence it is a favorable condition to perform high computation at node. Smart and efficient "store and forward" mechanism is used to buffer a packet at current node or to pass it to the next node. All discussed characteristics of VANET are mainly connected to single common issue that design of smart and efficient routing protocols.

Applications of VANET can be real time traffic analysis, route decision in case of emergency, entertainment systems, electronic toll collections, parking availability, post-crash notifications; road hazards control notification, remote vehicle personalization or diagnostic, availability of internet connection in some area and many more potential applications.

Proposed technique focuses on the issues of designing efficient and smart routing protocol using heuristics. A lot of work has been observed on mentioned issue, there are many heuristics used in literature for routing, proposed technique tried to merge these parameters and apply probabilistic and heuristic approach for making smart decision for routing in VANET [4] [5] [6]. Every node must broadcast their positions to its neighbor and hence it can maintain local routing information with every node. This information will be used for making routing decisions. Machine learning techniques i.e. probabilistic approaches for predicting behavior of traffic in some geographical location will be incorporated in future.

Paper is organized as follow, Section II describes the literature review, Section III describes characteristics of VANET, Section IV describes pros and cons of some of the protocols proposed in VANET, Section V describes Open Street Map (OSM), Section VI describes proposed methodology (Different components of system, Algorithms and techniques), Section VII describes about the heuristics used in proposed methodology, Section VIII describes Applications of proposed technique, Section IX describes theoretical results obtained, Section X explains the conclusion and future work has been discussed in Section XI. 


\section{RELATED WORK}

Several protocols has been discussed in [7] based on Geographic position of vehicles named as GR (geographic routing) or position based routing protocol (PBR) and shown these protocols very adequate in VANET due to robustness in dynamic environment. These protocols do not exchange link state information. The pros and cons of each of the GR protocol has been discussed.

Due to problem of connectivity in vehicular network, various parameters such as speed, density, direction and intervehicular distance for measurement of connectivity have been discussed in [8].

As there are various challenges in VANET such as high node mobility, changing topology, links breakages, constancy of networks and scalability of network. ANTSC protocol has been proposed that results in improved routing by considering various parameters such as speed differences between vehicles, direction, connectivity level and node distance etc. Proposed protocol in [9] is more scalable, robust and stable than existing one.

In [10] a message passing mechanism has been proposed for vehicular network.

Authors in [11], have a survey study on comparison of various protocols used for routing in vehicular ad-hoc networks. A comparison has been done on the basis on various parameters such as scalability, robustness, quality etc.

Authors in [12] highlighted the problem of designing routing algorithm for Ad-hoc VANET due to dynamic topology. Stimulated Annealing, clustering [13] [14], neural network using radial basis function are used considering many parameters such as velocity, free buffer size, etc. Simulations show the proposed performs outstanding in term of discovery rate and packet delivery rate.

Routing algorithm being a problem of finding shortest path, and that path should be optimized. Many of the optimized algorithms such as Ant Colony Optimization Algorithms, AOMDV, CRLLR, AQRV and T-AOMDV Algorithms are compared with proposed technique known as CRLLR (Clustering Based Reliable Low-latency Routing) in [15]. Parameters used for comparison used in that paper are throughput, Reliability, E2E Latency, Average Energy Consumption and Number of Beacons Messages. Considering these parameters two different experiments were performed.

1) Experiment A: The Impact of different relative velocities of vehicles having speed range between $40 \mathrm{~km} / \mathrm{hour}$ to $120 \mathrm{~km} /$ hour over the performance of CRLLR.

2) Experiment $B$ : The impact of different of different variety of vehicles on roads ranging from 30 vehicles to 100 vehicles to measure the significance of the proposed technique CRLLR, where it has been observed the average velocity of the vehicles is 60 kilometer per hours.

\section{Characteristics of VANET}

Vehicular Ad-hoc Network VANET holds some unique characteristics which make it distinguish from others networks such as MANET. These characteristics make it difficult and challenging to design VANET applications [16]. Following are the characteristics; one can target one of the characteristic and design routing protocol for that.

\section{A. High Dynamic Topology}

Vehicle moves at high speed up to $120 \mathrm{~km} / \mathrm{hr}$., which results different topologies at different time. So the topology is dynamic i.e. it changes with respect to time. Consider two vehicles are moving at the speed of $30 \mathrm{~m} / \mathrm{sec}$ and the radio range between them is $120 \mathrm{~m}$. Then the link between the two vehicles will last $120 / 30=4$ seconds that is vehicles are connected just for 4 seconds.

\section{B. Frequent Disconnected Network}

There is a chance of network disconnection between nodes i.e. vehicles, due to dynamic topology. One can have connectivity for very small duration i.e. connection period for information exchange may be for a very short interval. For sparse network this problem will be frequent.

\section{Mobility Modeling}

Mobility pattern of vehicles (nodes) highly dependent on road structure, speed of vehicles, driver's driving behavior, traffic environment. The geographic location also play vital role in designing routing protocols.

\section{Battery Power and Storage Capacity}

As compared to Mobile Ad-hoc NETwork there is enough computing power [11], battery power and storage. This enables us to work with effective communication and complex routing decisions.

\section{E. Communication Environment}

Environment of communication between vehicles is different in different networks i.e. urban environment and rural environment which can also be classified as sparse network and dense network. In those environments such as villages, cities, etc. [17]. there are building, trees and other objects behaves as obstacles and on highways these obstacles are less or even absent, so design of routing protocols are different according to the environment.

\section{$F$. Interaction with on-Board Sensors}

GPS plays important role in most of the routing protocols' design. Since the current position and movement of vehicles can be easily sensed by GPS. It will help us to design effective routing protocols.

\section{Existing Protocol Pros AND Cons}

Existing routing protocols are broadly classified into three different categories such as Pro-active Routing Protocol, Reactive Routing Protocols and Geo-graphic Routing Protocols. A node contributing in pro-active routing protocol maintains one or many tables representing the entire network topology, these routing tables are updated regularly for up-to date routing information from other nodes. Reactive routing protocol does not keep information of the entire network, it compute route to destination first while geographic routing protocol are based on the geographic locations of nodes [18] [19] [20]. 


\section{A. Fisheye State Routing}

FishEye State Routing Protocols is a proactive routing protocol (also known as Table driven protocol) as discussed in. Table driven protocols are where every node collects information from neighboring nodes. After collecting information, routing table is calculated. It is based on the link state routing \& an improvement of Global State Routing. Mentioned Algorithm has variety of pros and cons [21].

Pros:

- FSR exchanges partial update information with their own neighbor only, so reduce consumed bandwidth, hence results reduction in routing overhead.

- Reduce routing overhead.

- Link failure doesn't results changes in routing table.

Cons:

- Not performed well for small ad-hoc network.

- Less knowledge about distant nodes.

- Storage complexity and processing overhead is proportional to increase in network size.

- Insufficient information for route establishing.

B. Ad-Hoc on Demand Distance Vector (ADOV)

ADOV described in [22] stands for Ad-Hoc On Demand Distance Vector routing protocol, a reactive routing protocol which only establish a route when a node requires to send data packets to other nodes. It is capable of unicast and multi-cast routing.

Pros:

- An up-to-date path to the destination because destination sequence

- Reduces excessive memory requirements and redundancy.

- AODV responses to the link failure in the Network.

- It can be applied to large scale ad-hoc network.

Cons:

- Require more time connection setup \& initial communication to establish a route.

- It leads to inconsistency in case of out dated information at intermediate nodes.

- Control overhead will be heavy as there are multiple route reply packets.

- Consuming extra or excess bandwidth due to periodic and frequent beaconing.

\section{Temporally Ordered Routing Protocol (TORA)}

Temporally Ordered Routing Protocol uses Directed Acyclic Graph (DAG) concept for broadcasting packets. Here link reversal algorithm is used that creates a DAG (A directed graph that has a topological sorted order, a sequence of the vertices' such that every edge is directed from earlier to later in the sequence) towards destination, here source node act as a root of the tree.

Pros:

- It creates DAG (Direct acyclic graph) upon requirements.

- Intermediate nodes does not rebroadcast the messages, hence reduce network overhead.

Cons:

- DSR and AODV performed well as compared to TORA

- TORA is not scalable Routing Technique

\section{Geographic Routing Protocol (DTN)}

Geographic routing proposed in [7] is a routing that each node knows its own \& neighbor node geographic position by position determining services like GPS. It doesn't maintain any routing table or exchange any link state information with neighbor nodes. Information from GPS device is used for routing decision.

Pros:

- Route management and route discovery is not needed

- Scalable routing technique

- Performed very well for high node mobility pattern

Cons:

- It is based on GPS, hence it will not work in places where GPS services are not available.

\section{E. Delay Tolerant Networks}

Delay Tolerant Network (DTN) analyzed in [23], another routing technique uses carry and forward strategy in order to reduce frequent disconnection of nodes in the high mobility nodes network. Whenever a node is not in the contact with other node(s), then it store packet for some time on the basis of some defined metric and then on fulfilling some requirements it forward packet(s).

\section{OPEN STREET MAP}

Open Street Map, OSM is a map of the world, free to use under an open license. This is off-line version of map. API (Application Programmer Interface) is available in many different languages. OSM provide us details of the location like source to destination path, road types, junction etc. OSM uses A* Search Algorithm to find shortest path from source to destination. In $A^{*}$ Search the path is selected on the basis of following evaluation function

$f(n)=g(n)+h(n)$

Where $g(n)$ is the cost to next neighbor and $h(n)$ is the heuristic function, cost from neighbor to destination usually calculated by Euclidean distance formula, given by

$d=\sqrt{\left(x_{2}-x_{1}\right)^{2}+\left(y_{2}-y_{1}\right)^{2}}$ 
Here $\mathrm{x}_{2}, \mathrm{y}_{2}$ and $\mathrm{x}_{1}, \mathrm{y}_{1}$ are the latitude and longitude of two points in a path that is road.

\section{Methodology}

This proposed technique can be used in three different applications. One of the applications of proposed technique is emergency applications of VANET in a high dense urban area as shown in Fig. 1, where there are a lot of junctions and high traffic flow. It is difficult for ambulance or fire brigade ambulance to choose optimize route (typical use case from the situation shown in Fig. 1). The ambulance has to decide an optimize route. One cannot rely on straight forward route decided at start, because the traffic pattern is highly dynamic in urban areas, so it may change in small interval of time. OSM provides details of roads and their type's i.e. highway, motorway, etc. OSM has predefined junctions; junction can be view as a point where more than one road meets. The ambulance starts its journey from source point. It reads the first junction from OSM, then forwarded packet to know about the state of junction. The packet will be transfer to best neighbor among node and so on, at last the nodes available at junction (first node at some predefined radius) will reply broadcast message to all neighbors in its range and all neighbors will reply back to that node and then this node will respond back with traffic status (either yes or no and their speed, their own destinations) back to sender node. Hence it can show real time traffic information over off-line map services like OSM. This node will collect all information from nodes at junctions and will do some processing whether this route is good or not? And this information will be sent back to initial sender i.e. Ambulance. This node will also try to predict the current position of Ambulance using available information.

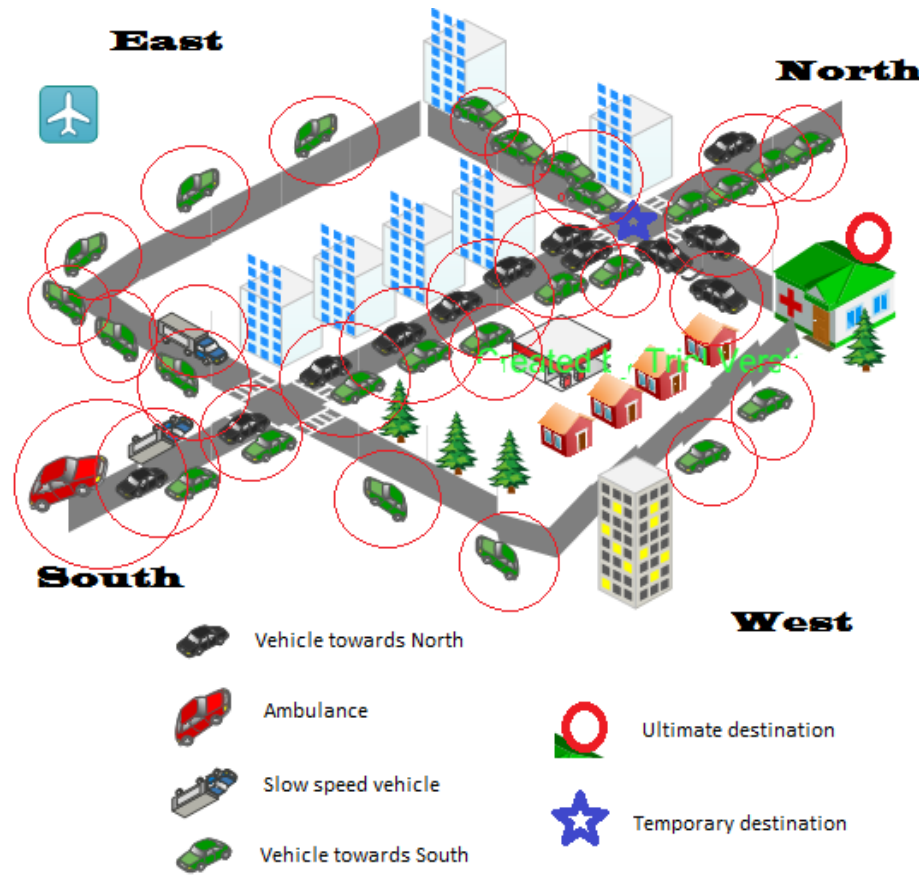

Fig. 1. Urban Area, Range of Vehicle Shown.

\section{Sender Packet Information}

Following information are sent from source to node available near destination.

1) Sender_ID

2) Sender_Geo_Location (using GPS)

3) Speed_of_Ambulance

4) Direction_of_Ambulance

5) Destination_address

6) Current_time_stamp

7) Road_Status_value

Where

- Sender_ID is the Global Identifier of source node; Sender_Geo_Location is the position (Latitude and Longitude) by the GPS services of Vehicle.

- Speed_of_Ambulance is the current speed of Source Node (Ambulance), which can be read by the speed meter device installed in vehicle.

- Direction_of_Ambulance can also be calculated at source node by observing successive GPS positions. Proposed technique considers only four directions North, West, East and South.

- Destination_address is the Address of Location about which one is interested to know the status; in this case it is the real time traffic situations in urban areas.

- Current_time_stamp is the time interval at which source node is sending packet to other node(s).

- Road_Status_value is an integer value initially set to zero when sending packet from source node.

Every node (nodes not near junction) that is forwarding this packet just increment this value by 1 .

\section{Intermediate Nodes Operations}

All the nodes that are just forwarding the packets with little update in packets are defined Intermediate Nodes. Every node when receives packets it check whether it should broadcast or not by comparing its own current position with destination address in packet.

\section{Algorithm at Intermediate Nodes:}

1 ...... Read destination address from packet

2.......Read its own Position

3...... Difference $=$ Position_current - Destination

4 .......if Difference $<=$ someRange

4.1.........CollectInformationFromNodes()

5 .......else

$5.1 \ldots . . .$. Read Road_Status_value from packet

$5.2 \ldots \ldots \ldots$.Increment this value by 1

Collect information method is only executed at the node, which is in the range of destination. This method can be scripted as given below: 


\section{First Node near destination}

The first encounter node, near destination will broadcast message to all neighbor nodes, and all neighbor nodes near that nodes (radius must be defined) will reply back by giving following information:

1) Traffic status (with yes or no, 1 or 0 bit)

2) Speed of the vehicle

3) Destination of each vehicle

Here, the intruder nodes are not taken into account, which may occurs some nodes can produce false information and may change the content of packet. In this technique no encryption or authentication schemes has been considered. It is considering all nodes to real and authentic nodes. The security aspect of this technique will be considered in future work. The Node processes this information will convert these into insights like the traffic congestion, time to clear, number of vehicles presents at junction, where there can be a possibility of congestion. The node will then create a response packet and send to that node that is Ambulance. The main problem is now what should be location/position of ambulance. Position, distance and location can be calculated by formulas mentioned below:

position $_{\text {initial }}=$ Location $_{\text {source }}$

distance $_{\text {covered }}=$ Speed $_{\text {source }} *\left(\right.$ time $_{\text {current }}-$ time $\left._{\text {source }}\right)---$

Location $_{\text {current }}=$ Location $_{\text {source }}+$ distance $_{\text {covered }^{--}}$

The intermediate node also known as Helper Node will also calculate where the next possible traffic is jam by calculating usage of roads. Helper node will find all the roads and their usage using the destination of all nods there at junction.

\section{Algorithm CollectInformationFromNodes:}

Once it is detected that node in the range of destination, it will execute following algorithm's steps

1...Broadcast Hello-beacon to all nodes in range

2 ...Every node at junction send Beacon-Reply

3...Add Length (all nodes that replied) to

Road_Status_value

4...Find Average Speed of nodes at junction

5...calculateTimeToClear()

6...predictCurrentPositionOfSender()

7...findRoadStatus()

8 ...createPacket()

9... Send Packet to Original Sender

Hello-beacon: Contains hello message

Beacon-Reply: Contains speed, destination address

\section{Algorithm predictCurrentPositionOfSender:}

$1 \ldots$ speed $=$ Read speed from Sender Packets

$2 \ldots$ position $=$ Read position from Sender Packets

3 ...direction $=$ Read direction from Sender Packets

4...time sender $=$ Read time stamp from from Sender Packets

$5 . .$. timeSinceThen $=$ Time $_{\text {sender }}-$ Time $_{\text {current }}$
6...Calculate distance covered till packet sent by using speed

.....equation, distance $=$ speed $*$ timeSinceThen

$7 \ldots$ position $=$ position + distance $($ covered in direction $)$

8.....return predicted position

\section{Algorithm findRoadsStatus:}

Static: Dictionary

1 ..... for all nodes at junctions

$1.1 \ldots \ldots . .$. road_id = findRoad(destination)

1.2 ........if road id not in Dictionary

$1.2 .1 \ldots \ldots \ldots \ldots$ Add road_id and count $=1$

1.3 ........else

$1.3 .1 \ldots \ldots \ldots \ldots$... Increase road count by 1

1.4 .......return Dictionary

createPacket is the name of function, which implements the computation of creating packets, it do all necessary steps before forwarded by helper node.

\section{Algorithm createPacket:}

Helper Node collects all the information calculated by previous methods and then sends back to Original Sender, in our case it is Ambulance. In this packet it will change source address to junction/location address and the destination address is set to the next predicted address of Original Sender i.e. Ambulance.

Helper node will create a packet having following information:

1) Source_address

2) Destination_address

3) Road_Status_value

4) Congested_Roads_informations

5) Time_to_Clear

\section{Main Algorithm Mode 1:}

1...if there are nodes in the range of Ambulance

1.1 .....Send Request-Beacon to all nodes

1.2.....All nodes returns Response-Beacon

1.3.....If there are all 0's in Response-Beacon

1.3.1......wait() and go to step 1

$1.4 \ldots . .$. else

1.4.1......packet sent successfully, wait for response

$2 \ldots$ else

2.1.....Keep packet until node appear in range

Request-Beacon: Contains Initial Packets discussed under heading Sender Packet Information (SPI).

Response-Beacon: If value is 1 , it means packet is send forwarded and discarded packet in case of value 0 .

\section{Main Algorithm Mode 2:}

1...if there are nodes in the range of Ambulance

$1.1 \ldots .$. Send Request-Beacon to all nodes

1.2.....All nodes returns Response-Beacon

1.3.....if direction is opposite of destination

$1.3 .1 \ldots . .$. .remove that nodes

1.3.2......Rem_Nodes are remaining Nodes

1.3.3.......Select best node amongst from Rem_Nodes 
1.3.4.......Forward packet to that node

1.3.5.......wait() for response

2 ...else

2.1....Keep packet until node appear in range

Request-Beacon: Contains Request Hello Message

Response-Beacon: Contains following information,

1) Speed

2) Direction

3) Buffer size

4) Range

5) GPS Position

\section{HEURISTICS USED IN METHODOLOGY}

In case of more than one available nodes i.e. vehicles which is a most obvious situation in urban dense area, multilane roads, it is necessary to pass packet to only node which is best node, to avoid broad-cast overhead. In order to find the best candidate node available, there are certain criteria like speed of vehicle, distance between ambulance and neighbors, distance between nodes and destination, buffer size, range of vehicle which is given below.

$f(n)=S+R+B+\left(\frac{1}{g(n)+h(n)}\right)---$

Where $f(n)$ is the cost of node, this value will be used for selecting best node for transmitting packet, $g(n)$ is the distance between Ambulance and Neighbor, $h(n)$ is the distance between node and destination, $\mathrm{S}$ is the speed of vehicle (node), $\mathrm{R}$ is the range of the vehicle (node), $\mathrm{B}$ is the size of buffer that vehicle have, $g(n)$ and $h(n)$ will be calculated by Euclidean distance formula given in equation (2). Select Node having large $f(n)$ Value and forwarded packet to that node.

Logic behind heuristic function: A node having larger buffer, high speed, large range, node nearest to ambulance and destination is good. High speed vehicle is good to deliver packet early and quick. Long range nodes are good to deliver packet from large distance. High buffer size nodes are good to select to keep packet(s) for long time.

Weighted heuristic function for selecting best node can be used as distance from node to destination and from source (ambulance) has very less impact, so we can assign low weight to this using following equation.

$f(n)=W_{1}(S+R+B)+w_{2}\left(\frac{1}{g(n)+h(n)}\right)$

Where $\mathrm{w}_{1}$ and $\mathrm{w}_{2}$ are the weights we can assign, but keep in mind that $\mathrm{w}_{1}+\mathrm{w}_{2}=1$, for example $\mathrm{w}_{1}=0.8$, then $\mathrm{w}_{2}=0.2$

If $\mathrm{w}_{2}=0$, and $\mathrm{w}_{1}=1$, it means you are totally ignoring $\mathrm{g}(\mathrm{n})$ and $\mathrm{f}(\mathrm{n})$, then equation will look like following

$$
f(n)=S+R+B
$$

\section{OTHER APPLICATIONS}

Apart from above discussed technique for specialized purpose, we can also modify this technique for following purposes, like
1) Entertainment Purpose: A song in one vehicle listens by another person in vehicle. Or we can even use it to play game between different vehicles over the network.

2) Emergency Purpose: Same as case given in this paper, this can be used for Ambulance, School Vans, Fire Fighter Ambulance or in case of emergency like accidents.

3) Location Analysis: To know about some fixed entity at some remote location like pizza shop, mall, item order etc. for example ordering something before you arrive at any point.

4) Real Time Traffic Analysis: This technique can be modified to analyze real time traffic pattern in some urban area as shown in Fig. 1 above. This will help us to find optimize routes in case of any emergency, accidents, war, or any natural disaster.

\section{RESULTS}

In proposed technique there is no broadcast overhead as only Helper node process information, all other nodes are free from sending broadcasts. Connection establishment is not required so as connection termination. Low processing, i.e. computation is carried out at some intermediate vehicles but not having redundant and waste processing on different nodes. Memory efficient Routing Protocol, i.e. no routing table or information is maintained at intermediate nodes. The proposed technique overcome problem discussed in DTN, that is problem of tracking locations where GPS is not working by using off-line maps such as OSM.

\section{COnClusions}

Efficient routing protocols can be designed using different heuristics like the position, speed, and direction of vehicles. In vehicular networks nodes can be having excess of energy and computation power, so we have to just take care of the delay and packet delivery of networks. Due to high mobility of nodes it was difficult to use a single parameter like speed or position. The use of off-line maps services like OSM, we can design best routing protocols. But still there is a lot of gap or research area to be work on these protocols designs. Store and forwards mechanism uses little bit intelligent approach. We have successfully shown on demand store and forward mechanism. Various applications of VANET has been discussed in this paper. Applications are emergency applications, security applications, entertainment applications and routing applications. Proposed system has not been proven effective by simulation and we are using heuristics which can misguide the routing process. These limitations are will be considered in future work of proposed system.

\section{FUTURE WORK}

Bio-inspired Algorithms and Machine Learning Algorithm can be used predict the pattern of traffic within some urban environment, so that routing decisions can be made properly and efficiently that will be incorporated in proposed technique in future. There is issue of security, any node if change packet content, will destroy whole communication and will leads to unreliable communication and process. A new technique can be designed in future by the name of Geo Encryption Technique, which will be location based encryption and decryption. 


\section{REFERENCES}

[1] Jaap, Sven, Marc Bechler, and Lars Wolf. "Evaluation of routing protocols for vehicular ad hoc networks in typical road traffic scenarios." Proc of the 11th EUNICE Open European Summer School on Networked Applications (2005): 584-602.

[2] Lin, Yun-Wei, Yuh-Shyan Chen, and Sing-Ling Lee. "Routing protocols in vehicular ad hoc networks: A survey and future perspectives." J. Inf. Sci. Eng. 26, no. 3 (2010): 913-932.

[3] Chekima, Ali, Farrah Wong, and Jamal Ahmad Dargham. "A Study on Vehicular Ad Hoc Networks." In 2015 3rd International Conference on Artificial Intelligence, Modelling and Simulation (AIMS), pp. 422-426. IEEE, 2015.

[4] Al-Kharasani, Nori, Zuriati Zulkarnain, Shamala Subramaniam, and Zurina Hanapi. "An efficient framework model for optimizing routing performance in VANETs." Sensors 18, no. 2 (2018): 597.

[5] Masegosa, Antonio D., Enrique Onieva, Pedro Lopez-Garcia, and Eneko Osaba. "Applications of soft computing in intelligent transportation systems." In Soft Computing Based Optimization and Decision Models, pp. 63-81. Springer, Cham, 2018.

[6] Toulni, Hamza, and Benayad Nsiri. "Cluster-based routing protocol using traffic information." International Journal of High Performance Computing and Networking 11, no. 2 (2018): 108-116.

[7] Boussoufa-Lahlah, Souaad, Fouzi Semchedine, and Louiza Bouallouche-Medjkoune. "Geographic routing protocols for Vehicular Ad hoc NETworks (VANETs): A survey." Vehicular Communications 11 (2018): 20-31.

[8] Hassan, Ahmed Nazar, Omprakash Kaiwartya, Abdul Hanan Abdullah, Dalya Khalid Sheet, and Ram Shringar Raw. "Inter Vehicle Distance Based Connectivity Aware Routing in Vehicular Adhoc Networks." Wireless Personal Communications 98, no. 1 (2018): 33-54.

[9] Mehmood, Amjad, Akbar Khanan, Abdul Hakim HM Mohamed, Saeed Mahfooz, Houbing Song, and Salwani Abdullah. "ANTSC: An intelligent Naïve Bayesian probabilistic estimation practice for traffic flow to form stable clustering in VANET." IEEE Access 6 (2017): 44524461 .

[10] Singh, Vijander, and G. L. Saini. "Dtn-enabled routing protocols and their potential influence on vehicular ad hoc networks." In Soft Computing: Theories and Applications, pp. 367-375. Springer, Singapore, 2018.

[11] Kumar, Rakesh, and Mayank Dave. "A comparative study of Various Routing Protocols in VANET." arXiv preprint arXiv:1108.2094 (2011).
[12] Bagherlou, Hosein, and Ali Ghaffari. "A routing protocol for vehicular ad hoc networks using simulated annealing algorithm and neural networks." The Journal of Supercomputing (2018): 1-25.

[13] Toulni, Hamza, and Benayad Nsiri. "Cluster-based routing protocol using traffic information." International Journal of High Performance Computing and Networking 11, no. 2 (2018): 108-116.

[14] Khan, Ammara Anjum, Mehran Abolhasan, and Wei Ni. "An evolutionary game theoretic approach for stable and optimized clustering in vanets." IEEE Transactions on Vehicular Technology 67, no. 5 (2018): 4501-4513.

[15] Abbas, Fakhar, and Pingzhi Fan. "Clustering-based reliable low-latency routing scheme using ACO method for vehicular networks." Vehicular Communications 12 (2018): 66-74.

[16] Agarwal, Pallavi. "Technical review on different applications, challenges and security in VANET." Journal of Multimedia Technology \& Recent Advancements 4, no. 3 (2018): 21-30.

[17] Rana, Kamlesh Kumar, Sachin Tripathi, and Ram Shringar Raw. "Analytical Analysis of Improved Directional Location Added Routing Protocol for VANETS." Wireless Personal Communications 98, no. 2 (2018): 2403-2426.

[18] Paul, Bijan, Md Ibrahim, Md Bikas, and Abu Naser. "Vanet routing protocols: Pros and cons." arXiv preprint arXiv:1204.1201 (2012).

[19] Singh, Gagan Deep, Ravi Tomar, Hanumat G. Sastry, and Manish Prateek. "A review on VANET routing protocols and wireless standards." In Smart computing and informatics, pp. 329-340. Springer, Singapore, 2018.

[20] Krishnan, V.G. and Sankar N.R., ICR: information, cluster and route agent based method for efficient routing in VANET. International Journal of Engineering \& Technology 7(2018), no. 1.9 (pp10-15)

[21] Pei, Guangyu, Mario Gerla, and Tsu-Wei Chen. "Fisheye state routing: A routing scheme for ad hoc wireless networks." In 2000 IEEE International Conference on Communications. ICC 2000. Global Convergence Through Communications. Conference Record, vol. 1, pp. 70-74. IEEE, 2000.

[22] Kohli, Sandhaya, Bandanjot Kaur, and Sabina Bindra. "A comparative study of Routing Protocols in VANET." Proceedings of ISCET (2010).

[23] Raw, Ram Shringar, and Arushi Kadam. "Performance Analysis of DTN Routing Protocol for Vehicular Sensor Networks." In Next-Generation Networks, pp. 229-238. Springer, Singapore, 2018. 